\title{
CT Evaluation of Temporal Bone Malignancies
}

\author{
Raini K.P ${ }^{1}$, M. N. Brahmadathan ${ }^{2,}$ K.Mohanan ${ }^{3}$, Suny Thomas ${ }^{4}$, Mini .M.V ${ }^{5}$, \\ Elizabeth Daniel $^{6}$, Paul V Puthussery ${ }^{7}$ \\ 1, 5, 6,7 (Assistant Professor, Dept. of Radiodiagnosis, medical college, Thrissur, Kerala, India) \\ 2, 3,4 (Professor, Department of Radiodiagnosis, Medical College, Thrissur, Kerala, India.)
}

\begin{abstract}
:
Introduction: High Resolution Computed Tomography (HRCT), a modification of routine CT, provides a direct visual window in the temporal bone providing minute structural details. Purpose of the present study was to evaluate the normal variations, pathological processes and their extent involving the temporal bone along with their complications on HRCT and to correlate these imaging findings surgically, wherever available. Methodology: The prospective study included 30 patients who were referred to the radiology department with clinically suspected temporal bone or ear pathologies. After detailed clinical examination, the patients were subjected to high resolution computed tomography (HRCT) examination. The imaging findings were correlated with the surgical findings wherever available. The surgical findings were considered as final.

Results: From a total of 30 cases, $40 \%$ had acoustic neuroma. The surgical and radiological findings showed a high level of sensitivity (95\%) in the identification of acoustic neuroma. HRCT provides a good sensitivity of 93.3\% in the identification of changes to the ossicular chain despite the presence of surrounding soft tissue. HRCT was highly informative in identification of erosion of lateral semicircular canal. In the evaluation of any congenital abnormality of the ear HRCT proved to be beneficial in depicting the anatomical details. Conclusion: The clinical and radiological findings showed a high level sensitivity with intra operative findings as regards to the presence of acoustic neuroma, changes of the internal auditory canal and erosion of the lateral semicircular canal. HRCT findings, in the treatment of any congenital abnormality of the ear were a good guide to the surgeon for planning and management.
\end{abstract}

Keywords: Acoustic neuroma, High Resolution Computed Tomography, Meningioma, Temporal bone.

\section{Introduction}

The tympanic cavity is an air-containing space within the temporal bone, which communicates with the nasopharynx through the Eustachian tube and with the mastoid air cells by means of the tympanic antrum. It constitutes an extension of the upper respiratory tract and is subject to viral and bacterial invasion through Eustachian tube.

A major advance in diagnostic imaging has occurred with the introduction of High Resolution computed Tomography which have made it possible to obtain high quality images with exquisite demonstration of most normal temporal bone structures and numerous pathological processes. High resolution computed tomography provides excellent of bony land marks within the temporal bone, due to the temporal bone inherent contrast, its dense bone being surrounded by air of the tympanic cavity and mastoid air cells. It has also added whole new dimension to the temporal bone by allowing visualization of the of the tissue components within and adjacent to the temporal bone. Because high resolution computed tomography can assess this area with unprecedented accuracy, it has allowed better understanding of the etiology, pathology, the disease course earlier detection of complications and treatment modality which has considerably reduced the morbidity and mortality pertaining to lesions of this region.

\section{Aims \& Objectives.}

To assess the role of High Resolution Computer Tomography as the prime modality in the diagnosis and characterization of lesions of the temporal bone

\section{Materials and Methods.}

The ethics committee of our institute approved this prospective study and an informed consent was taken from all patients included in it. The prospective study included 30 patients with clinically suspected temporal bone or ear pathologies seen at Govt. Medical College, Thrissur, Kerala, India. However patients with history of trauma and those with known or detected neoplasm related to temporal bone were excluded.

All the patients underwent a detailed clinical ENT examination followed by HRCT temporal bone. They were evaluated with Multidetector Computed Tomography technique of inner ear in the SIEMENS SOMATOM EMOTION 16 CT machine. No financial burden was incurred on the patient. 
Scout films are taken routinely in all patients before starting the scan. Scans were acquired in the helical mode to reduce motion artifacts. With the patient in supine position \& slight extension of the head, axial projections were obtained by serial 1-2 $\mathrm{mm}$ thin sections of the temporal bone with the line joining the infra-orbital rim and external auditory meatus perpendicular to the table. Scanning parameters of $133 \mathrm{kV}, 140 \mathrm{mAs}, 1-2 \mathrm{~mm}$ section thickness, $0.6 \mathrm{~mm}$ collimation were taken. The images were reconstructed with a bone algorithm. Coronal and sagittal reformatting was done to a slice thickness of $0.75 \mathrm{~mm}$. intravenous contrast was administered to study the hyper vascular lesions like glomus tumors, cerebellopontine angle masses, intracranial or extra cranial extension of middle ear diseases.

Each HRCT image were analyzed for specific features relevant to the evaluation of pathologies of temporal bone and interpreted in detail to define: 1 The type, location and extent of lesion., 2. Bony erosions of middle ear walls. 3. The integrity of the ossicular chain, facial nerve canal and labyrinth. 4. Involvement of hidden area, mastoid air cell system.

The final diagnosis was made by medical, per operative and histopathological examination. All pathology reports were reviewed. The findings of HRCT were correlated with medical response, per operative finding and histopathologic diagnosis.

\section{Results}

Out of 30 included cases in the study, surgical exploration was done in all cases. Out of 30 cases studied, 18 cases $(60 \%)$ were males, $12(40 \%)$ females. In this study, the age range was 10-75. Seventeen patients (34\%) belong to $21-30$ years of age group. The most common presenting symptom was head ache. Table. 1 shows the presenting complaints of patients. The most common presenting symptoms were head ache $(80 \%)$, hearing loss $(60 \%)$, otalgia $(66.7 \%)$, vertigo/tinnitus (60\%), facial weakness (33.3\%), and cerebellar sign (26.7\%).

Among the 30 cases, Patients with acoustic neuroma form the largest proportion of cases, finding of majority of cases were suggestive of acoustic neuroma in $(n=15) 50 \%$ followed by meningioma in $(\mathrm{N}=5)$ $16.7 \%$, paraganglioma in $(\mathrm{n}=2), 6.7 \%$, External auditory canal malignancy in $(\mathrm{n}=2), 6.7 \%$, Right temporal region malignancy in $(n=2), 6.7 \%$ and metastasis in $(n=4), 13.3 \%)$. Table 2 shows the distribution of diseases.

30 cases of tumors involving temporal bone were studied. Table 3 describes the comparison of HRCT finding with biopsy finding of tumor cases. 15 were Schwanoma presenting as CP angle masses. All 4 were large masses causing widening of internal acoustic meatus and had an intracanalicular component. 3 were heterogeneous and were enhancing. 1 was homogenously enhancing. 3 patients were middle aged females and 1 was a middle aged male. These were 14 cases proved to be schwannoma on surgery and biopsy. 5 patients were diagnosed as meningiomas which were confirmed by surgery.

2 patients reported to have carcinoma of the external auditory canal with extensive bone involvement. Patients were confirmed as having squamous cell carcinoma on biopsy. They were referred to radiotherapy department for further management. Figure 1. Shows carcinoma of external auditory canal.

2 patients diagnosed as soft tissue malignancy of temporal region with extensive bone involvement. There was significant soft component which was enhancing too. Extensive bone erosion was also associated. But on biopsy one of the patient had cholesteatoma. These were confirmed during surgery.

\section{Discussion}

HRCT imaging is necessary for anatomic determination which acquiring an increasingly important role in the radiographic assessment of temporal bone. Radiographic assessment of temporal bone is difficult owing to complicated anatomical structure of middle and inner ear. This study is undertaken to develop a systemic method for evaluation of temporal bone as there are a variety of other imaging modalities. The lowest radiation dose to the lens, visualization of small bony structures, technical factors, ease of patient positioning, interpretation of the images and economical factors were all considered.

HRCT has the advantage of excellent topographic visualization, devoid of artifacts from superimposition of structures. It helps in accurate assessment of pathology prior to surgical exploration regarding location, extent and complication of the disease.

They constitute $12 \%$ of our study which is not correlated with the study of GAS Lloyd et al (1980) ${ }^{4}$ which claimed neoplasm to be the most frequent lesions. Age group of these patients in our series varied from 31 to 60 years with female preponderance. $2: 1$ mean age 47.8 .

H Imhof, CB Henk,AD Irisamer, C Czerny ${ }^{5}$ et al in 2003 published an article on CT characteristics of the tumors of the temporal bone and the cerebellopontine angle. HRCT as usually performed in the axial plane are using a high resolution bone window level setting, coronal planes are reconstructed from the axial data set or will be obtained directly. The study excellently depicts the osseous changes for example, the exostosis of the external auditory canal and which also useful in characterizing the lesion into benign or malignant. HRCT is an 
excellent method to depict and mostly characterize tumor lesions and helps in differentiating benign or malignant lesions. Also shall be used complimentary and have great impact in therapeutic planning.

Acoustic neuroma: Out of the 30 neoplastic lesions that were scanned 14 (93.3\%) were diagnosed as acoustic neuroma. Right CP angle predominant was noted in our study. All cases were hypo dense to hyper dense to the surrounding brain with good enhancement on contrast administration and depicted internal auditory canal widening, erosion and intra canalicular extension. No hyperostosis of adjacent bone noted.

Taylor S (1982) ${ }^{6}$, in his study had reported bony erosion on CT in up to $87 \%$ of the cases. This difference can be because we encountered all large size acoustic neuroma. Acoustic neuroma was the most common internal auditory canal and or CP angle lesions in a study by P Wolf (1987) ${ }^{7}$ and GAS Lloyd (1980) ${ }^{4}$.

Helie $\mathrm{O}$ et all had reported Meningioma are the second most frequent CPA mass, accounting for $10 \%$ to $15 \%$ of cases ${ }^{8}$,. On CT, approximately $20 \%$ will show calcification, and some may exhibit adjacent bone hyperostosis or enostotic spurs 9 .

The other tumor we studied on HRCT was carcinoma of the external auditory canal with extensive bone erosion. This was proved by biopsy as squamous cell type and the patient was sent to radiotherapy department for further management. Bird CR, Hasso AN, Stewart CE ${ }^{10}$ et al studied malignant primary neoplasm of the ear and temporal bone by HRCT and found this as a useful tool in delineating the extend of the tumor and bone destruction, which is still necessary for predicting out come and planning further management.

The third case was an extensive soft tissue lesion of the right temporal region with a extensive bone destruction which was diagnosed as a malignant lesion by HRCT due to the characteristics like size, margins, extensive involvement of adjacent structures and dense contrast enhancement. But on biopsy the patient was proved to have cholesteatoma with no evidence of malignancy in the specimen provided. This was probably a giant cholesteatoma ${ }^{11}$; one such is described in an article published by Ilhan TopaloGlu, MD, Hasan Tahsin cad. Fatma Hanim in SAGE journals.

Jahn, ${ }^{12}$ in a study of 11 patients, discussed the intratemporal spread of temporal bone metastases. Tumor emboli from a primary site such as the breast-lodged in the marrow-containing portion of the petrous bone, in the region of the internal auditory canal. The marrow spaces are subsequently infiltrated by tumor, followed by vascular extension into the mastoid air cells. In our study 4 patients (13.3\%) were diagnosed to have metastasis.

\section{Tables And Figures}

Table 1: shows the presenting complaints of patients in the study population.

\begin{tabular}{|l|l|l|}
\hline Clinical features & Frequency & Percentage \\
\hline Hearing loss & 18 & 60 \\
\hline Otalgia & 20 & 66.7 \\
\hline Vertigo/tinnitus & 24 & 80 \\
\hline Facial weakness & 10 & 33.3 \\
\hline Head ache & 22 & 73.3 \\
\hline Cerebellar sign & 8 & 26.7 \\
\hline
\end{tabular}

Table 2: Frequency of study population based on type of tumor.

\begin{tabular}{|l|l|l|}
\hline Type & Frequency & Percentage \\
\hline Schwannoma & 15 & $50 \%$ \\
\hline Meningioma & 5 & $16.7 \%$ \\
\hline Paraganglioma & 2 & $6.7 \%$ \\
\hline External auditory canal malignancy & 2 & $6.7 \%$ \\
\hline Right temporal region malignancy & 2 & $6.7 \%$ \\
\hline Metastasis & 4 & $13.3 \%$ \\
\hline Total & 30 & $100 \%$ \\
\hline
\end{tabular}

Table 3: Comparison of HRCT finding with biopsy finding of tumor cases.

\begin{tabular}{|l|l|l|l|l|l|l|}
\hline Type & CT cases & $\begin{array}{l}\text { Biopsy } \\
\text { Positive }\end{array}$ & $\begin{array}{l}\text { Biopsy } \\
\text { Negative }\end{array}$ & Percentage & Sensitivity & Specificity \\
\hline Schwanoma & 15 & 14 & 1 & $93.3 \%$ & 1.00 & 0.06 \\
\hline Meningioma & 5 & 5 & 0 & $100 \%$ & - & - \\
\hline Paraganglioma & 2 & 1 & 1 & $50 \%$ & 1.00 & 0.5 \\
\hline $\begin{array}{l}\text { External auditory } \\
\text { canal malignancy }\end{array}$ & 2 & 2 & 0 & $100 \%$ & - & - \\
\hline $\begin{array}{l}\text { Right temporal } \\
\text { region } \\
\text { malignancy }\end{array}$ & 2 & 1 & 1 & $50 \%$ & - & - \\
\hline Metastasis & 4 & 4 & 0 & $100 \%$ & - & - \\
\hline
\end{tabular}




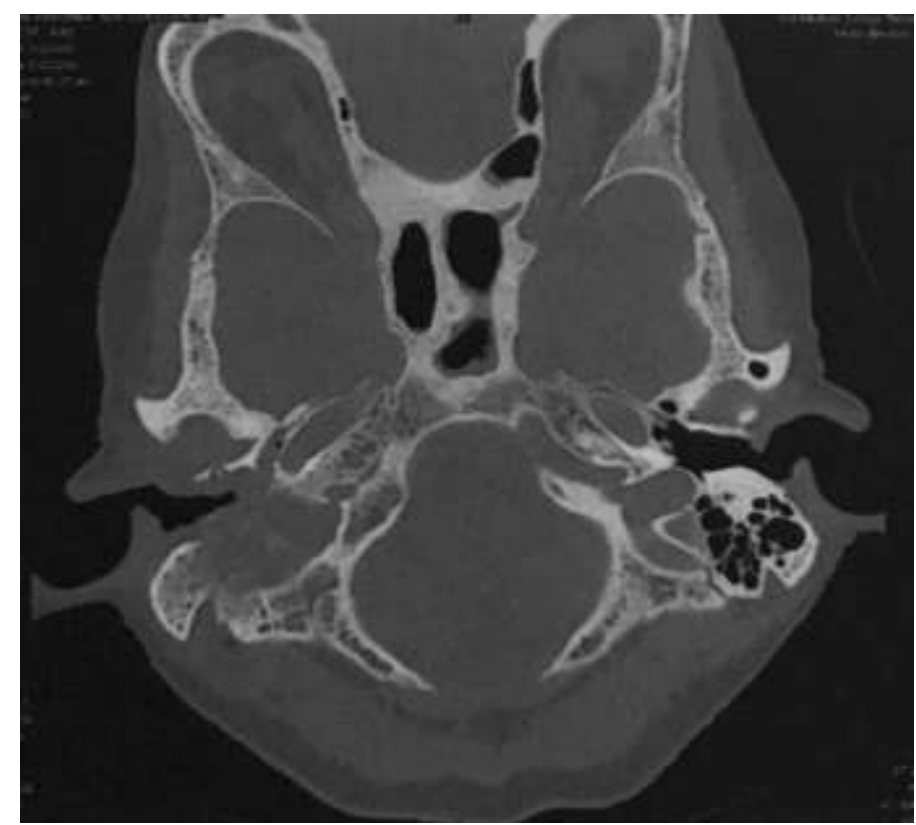

Figure 1: HRCT temporal bone axial section showing carcinoma of right external auditory canal with bone destruction.

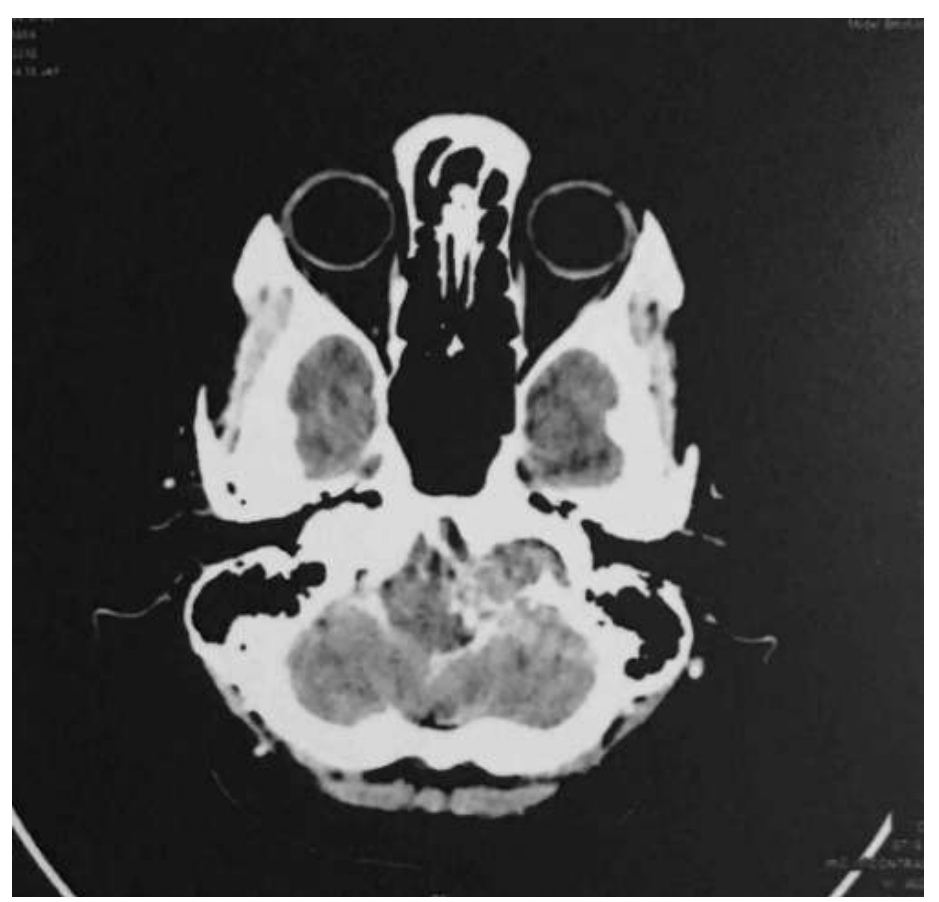

Figure 2: Post contrast CT axial sections showing left CP angle schwanoma with intra canalicular component.

\section{Conclusion}

Overall, the results of the present study showed a good agreement between HRCT and operative findings. HRCT temporal bone is an efficacious modality for accurate delineation of the anatomy and pathological involvement of temporal bone. We can hence conclude HRCT is useful for diagnosis, surgical planning and management of temporal bone pathologies.

\section{References}

[1]. Brogan M, Chakeres DW. Computed tomography and magnetic resonance imaging of the normal anatomy of the temporal bone. Semin Ultrasound CT MR. 1989; 1010:178-94.

[2]. Howard JD, Elster AD, May JS. Temporal bone: Three dimensional CT. Radiology. 1990; 177(2):427-30.

[3]. Chakeres DW, Augustyn MA. CT and MR Imaging of the Whole Body. 4th ed. Ohio: Mosby; 2003. Temporal bone. In: Haaga JR., Lanzieri CF, Gilkeson RC; pp. 495-512. 
[4]. Lloyds GAS, Phelps PD and Du Boulay GH. High resolution computerized tomography of the petrous bone. Brit. J. Radiology 1980; 53: 631.

[5]. CTcharacteristics of the tumors of the temporal bone and cerebellopontine angle. H Imhof ,C B Henk, A D Irisamer, C Czerny et al in 2003 radiology journal.

[6]. Taylor S. The petrous temporal bone (including the cerebello-pontine angle). Radiol Cling north Am 1982; 20: 67-86.

[7]. Allan P Wolff, Mikhael A MikhaelEvaluation IL and Ivan S Clric. Current concepts in neuroradiological diagnosis of acoustic neuromas. J Laryngoscope 1987 April; 97: 471-476.

[8]. Sarrazin JL. Infra tentorial tumors. J Radiol. 2006 Jun; 87(6 Pt 2):748-763.

[9]. Helie O, Soulie D, Sarrazin JL, Derosier C, Cordoliani YS, Cosnard G. Magnetic resonance imaging and meningiomas of the posterior cerebral fossa. 31 cases. J Neuroradiol. 1995 Dec; 22(4):252-270.

[10]. Bird C R, Hasso A N, Stewart CE, Malignant primary neoplasm of the ear and temporal bone by HRCT. Radiology 1983(1): 171174.

[11]. Giant Cholesteatoma Presenting as a Postauricular Mass Ilhan Topaloglu, MD 1 Mustafa Zafer Uguz, MD 1 Fazil Necdet Ardic, MD 1Department of Otolaryngology and Head Neck Surgery Clinic of Izmir Ataturk State Hospital. Iszm ir Turkey, sage journals online.

[12]. Jahn AF. Farkashidy J. Berman JM. Metastatic tumors in the temporal bone-a pathophysiologic study. J Otolaryngol 1979;8: I 